ÉGYPTE monde arabe

\section{Égypte/Monde arabe}

4 | 2007

Figures de la santé en Égypte

\title{
L'hépatite $C$ et les défaillances du système égyptien de santé public
}

Itinéraires thérapeutiques et solutions palliatives

\section{Saadia Radi}

\section{OpenEdition}

\section{Journals}

Édition électronique

URL : https://journals.openedition.org/ema/1766

DOI : 10.4000/ema.1766

ISSN : 2090-7273

Éditeur

CEDEJ - Centre d'études et de documentation économiques juridiques et sociales

Édition imprimée

Date de publication : 31 décembre 2007

Pagination : 127-145

ISBN : 2-6905838-43-4

ISSN : 1110-5097

\section{Référence électronique}

Saadia Radi, «L'hépatite $C$ et les défaillances du système égyptien de santé public », Égypte/Monde arabe [En ligne], 4 | 2007, mis en ligne le 31 décembre 2008, consulté le 07 juillet 2022. URL : http:// journals.openedition.org/ema/1766 ; DOI : https://doi.org/10.4000/ema.1766 


\title{
SAADIA RADI
}

\section{L'HÉPATITE C ET LES DÉFAILLANCES DU SYSTÈME DE SANTÉ PUBLIQUE}

\author{
ITINÉRAIRES THÉRAPEUTIQUES ET SOLUTIONS PALLIATIVES
}

\begin{abstract}
a maladie n'est pas seulement un désordre biologique qui atteint l'individu dans son corps et nécessite un recours thérapeutique faisant appel à un savoir médical. La maladie est aussi une réalité sociale qui interpelle l'entourage de la personne atteinte, affecté par la souffrance d'un proche. Elle met également en question l'économie et surtout le politique, tout spécialement lorsqu'il s'agit d'une épidémie. La maladie n'est alors plus une gestion individuelle du corps. Chez les anthropologues, la maladie a longtemps été étudiée selon deux angles souvent complémentaires : celui de sa représentation culturelle et des pratiques des thérapeutes et celui, plus spectaculaire, de la possession, la sorcellerie, de la divination et de les transes. Les « itinéraires thérapeutiques »- les choix successifs de cures opérés par le malade - ont souvent été abordé d'un seul point de vue : les malades se soignent selon leurs croyances et selon leurs représentations. Cette manière de voir les choses est insuffisante, parce qu'elle enferme le sujet dans un champ restreint, celui de sa culture.

Didier Fassin, dans L'Espace politique de la santé, soutient que :

dans toutes les sociétés la maladie met en jeu des rapports de pouvoirs.

Elle les exprime dans les corps à travers les différences entre les individus face aux risques de l'existence ou aux possibilités de se soigner, qui sont autant de façons d'inscrire physiquement l'ordre social. Elle les révèle dans l'intervention de ceux que l'on crédite de la capacité de guérir, qu'ils soient chamanes, marabout ou médecins, mais aussi dans les relations qui s'instaurent entre les professions de santé et les pouvoirs publics. (Fassin, 1996, p. 3)
\end{abstract}

Le même auteur propose de mettre la question du pouvoir au centre des études sur la santé et la maladie. Ce recentrage permet au chercheur d'échapper 
au découpage opposant les sociétés traditionnelles et les sociétés modernes, les pratiques du passé et celles du présent, et permet d'examiner conjointement des pratiques que I'on a l'habitude d'étudier séparément.

En effet, bien que la représentation de la maladie et la culture soient toujours des domaines pertinents pour l'anthropologie de la maladie, il ne faut désormais plus se contenter d'étudier la relation que la personne malade entretient avec sa culture mais aussi interroger les politiques publiques de santé et les relations que les malades entretiennent avec elles. II faut analyser et comprendre leurs recours et s'interroger sur ce que le système de santé public offre aux citoyens, sur les politiques de santé publique et sur la manière dont les malades sont traités dans les institutions qui en relèvent. Avec la globalisation, nous nous trouvons dans un "village planétaire ", où les différences en matière de santé et de maladie ne relèvent pas d'une culture locale mais de la position que chaque groupe occupe dans sa propre société, où comme l'explique Bernard Ours, "Le développement ne se pose plus comme un problème entre les nations mais entre les groupes sociaux dans le "village planétaire" » (Hours, 2001, p. 15). Un riche Cairote se soigne comme un riche Parisien ; il peut utiliser la médecine égyptienne comme il peut aller se soigner dans les meilleures cliniques parisiennes, s'il estime que la médecine égyptienne ne répond pas à ses attentes - et un Égyptien pauvre se soigne, lui, comme il peut.

Mon article porte sur la manière dont des Égyptiens atteints par le virus de I'hépatite $C$ se soignent. J'étudie en parallèle la manière dont ils le font et la politique égyptienne en matière de santé publique, en montrant le rôle crucial que celle-ci joue dans la structuration des itinéraires thérapeutiques. J'aborde aussi la responsabilité directe ou indirecte des gouvernants - c'est-à-dire du système de santé publique, tel qu'il est - dans la transformation de scientifiques, médecins ou vétérinaire, en thérapeutes néo-traditionnels, laissant par là même le champ libre à toutes sortes d'autres thérapeutes considérés, par le même système, comme des charlatans.

\section{L'HÉPATITE C EN ÉGYPTE}

L'hépatite C est une maladie virale du foie ; les porteurs du virus risquent de développer une affection hépatique chronique, une cirrhose et un cancer du foie. Le virus de I'hépatite C (VHC) a été isolé en 1989. Avant cette date, on parlait d' « hépatite non A non B ». Ce virus pose actuellement un considérable problème de santé publique. L'OMS estime que 170 millions de personnes seraient atteintes par le virus de I'hépatite C, soit $3 \%$ de la population mondiale, et que, chaque année, 3 à 4 millions de personnes seraient infectées (communiqué de presse de l'OMS du 14 mars 2002). Cette maladie existe partout dans le monde et sa prévalence varie selon les pays et les régions : elle est de 5,3\% en Afrique; de 1,7\% en Amérique ; de 4,6\% en Méditerranée 
orientale ; de 1,03 \% en Europe ; de 2,15\% en Asie du Sud-est ; et de 3,9\% dans le Pacifique occidental. Le VHC se transmet principalement par le contact direct du sang contaminé. Il peut infecter lors d'une transfusion sanguine, d'une dialyse ou par la réutilisation de seringues ou de matériel médical mal stérilisé. Il se transmet aussi, hors des milieux médicaux, par l'utilisation d'objets coupants contaminés, comme le matériel du coiffeur, celui du pédicure ou celui du manucure mais aussi lors des excisions et des circoncisions. II peut aussi se transmettre, dans de rares cas, par voie sexuelle. Jusqu'à présent, aucune autre cause de transmission n'a été observée. La particularité de cette maladie est le fait qu'elle est asymptomatique.

De nombreuses personnes atteintes par ce virus ignorent leur état et la plupart de celles qui savent qu'elles sont malades l'on découvert par hasard ou lorsque leur état s'est aggravé. C'est pour cela qu'en Égypte on nomme cette maladie la «maladie silencieuse ».

L'Égypte est le pays où la prévalence de l'hépatite C est la plus élevée au monde : elle serait de $13 \%$ selon l'OMS. La cause initiale de sa propagation résiderait dans la campagne de traitement de masse contre la bilharziose, menée entre 1960 et 1980, et impliquant des injections. Il est actuellement établi que les seringues utilisées durant cette campagne étaient mal stérilisées ou pas stérilisées du tout. Ceci expliquerait que le taux de prévalence de I'hépatite $C$ est très élevé chez les adultes de certaines zones rurales, âgés de plus de 40 ans. Selon I'Inserm, elle peut atteindre, dans certaines zones, jusqu'à 50 \% de la population. L'étude d'une cohorte dans un village du delta du Nil, dirigée par le Pr. Mustafâ K. Muhammad, de l'université de 'Ayyn Shams, au Caire, et le Pr. Arnaud Fontanet, de l'Institut Pasteur, a montré que :

La prévalence d'anticorps anti-VHC est de $20 \%$ chez les adultes, atteignant $50 \%$ chez les hommes qui ont plus de 45-50 ans (de l'ordre de $30 \%$ chez les femmes, le taux de scolarisation des filles au moment des campagnes de traitement de la bilharziose étant moins élevé). On retrouve des taux atteignant les 5 à $10 \%$ chez les 15 à 30 ans et des infections qui démarrent chez l'enfant, dès l'age de 5 ans. En terme d'incidence, on estime qu'il y a environ 3 nouveaux cas pour 1000 habitants par an. ${ }^{1}$

Les résultats de cette étude confirment donc que la première cause de la contamination par le VHC en Égypte réside bien dans la campagne de traitement contre la bilharziose - sans oublier d'autres causes, moins importantes mais probablement contemporaines, comme la scarification médicale, que I'on pratique par exemple pour le BCG. Même si la prévalence du VHC a baissé chez les classes d'âges n'ayant pas été concernées par cette campagne,

1. Source : "Particularité de I'hépatite C en Égypte », Actualité de l'Inserm, n²03, novembre 2006. 
elle reste encore très élevée par rapport à la prévalence mondiale. Comment peut-on expliquer que I'hépatite $C$ ait continué à se propager?

Avant la mise en évidence de l'agent viral de l'hépatite C, la presse égyptienne évoquait déjà l'existence de ce virus dans le pays en tant qu' « hépatite non A non B ». Durant la période 1989-1990, la presse a tenu un discours alarmant sur ce nouveau virus qui allait s'ajouter aux autres et « détruire le foie des égyptiens », déjà attaqué par la bilharziose et I'hépatite B. Ce discours était souvent accompagné de déclarations de médecins ou d'entretiens avec des spécialistes de la question comme le Dr. Yâsîn 'Abd al-Ghaffâr et le Dr. 'Abd al-Rahmân al-Zabadî. Cependant, le système de santé publique ne réagissait pas. Il fallut attendre 1995 pour que I'ampleur de l'épidémie soit officiellement reconnue. En 1994, 'Abd al-Ghaffâr fit une conférence en Arabie Saoudite où il exposa la gravité de la situation en Égypte. À la suite de cette conférence, des pays du Golfe exigèrent un certificat médical attestant que les Égyptiens candidats à l'immigration n'étaient pas atteints par le virus de I'hépatite C. Les autorités furent mises dans l'obligation d'agir, tant à la suite du scandale provoqué par la déclaration du médecin qu'à cause des exigences des pays destinataires de l'immigration égyptienne ${ }^{2}$.

La reconnaissance officielle de l'existence d'une maladie ou d'une épidémie est un moment crucial de la « carrière » de celle-ci, dans la mesure où elle ne devient un problème de santé publique que lorsqu'elle est un problème pour les instances politiques (Dodier, 2003). Tant qu'elle n'en est pas un, celles-ci ne font pas de prévention, ne limitent pas les moyens de transmission de la maladie et ne mesurent même pas son avancée. Tel était le cas de l'Égypte avant la reconnaissance officielle de l'épidémie ; c'est ce qui explique, au moins en partie, sa propagation après l'arrêt des injections et, surtout, après la mise en évidence du virus en 1989.

\section{LES FACTEURS DE TRANSMISSION DE LA MALADIE APRÈS LA CAMPAGNE D'INJECTIONS CONTRE LA BILHARZIOSE}

Aujourd'hui, il n'y a plus de doute sur le fait que les seringues non stérilisées, lors de la campagne contre la bilharziose, ont contaminé un noyau initial de personnes, mais que la contamination a continué à se propager au-delà des années quatre-vingt. Parmi les vecteurs de contamination cités, la transfusion sanguine occupe une place particulière et met particulièrement en cause les instances politiques.

À part en période de guerre, le pays n'a jamais réussi à avoir suffisamment de sang pour traiter les malades. L'Égypte importait donc une partie du sang destiné à la transfusion. Cependant, avec la découverte du virus du sida, elle arrêta

2. Pour un compte rendu circonstancié, voir Radi, 2006. 
cette importation par crainte de la contamination. Il y eut alors plusieurs tentatives pour sensibiliser la population au don du sang. Ainsi, un ministre de la santé est-il apparu à la télévision en train de donner son sang, l'épouse du président de la République et la Croix-Rouge ont-ils organisé une campagne nationale de don, en novembre 1997, au profit des enfants malades. Ces tentatives, et d'autres poursuivant le même but, n'ont pas abouti à des résultats significatifs. Les banques de sang, qu'elles soient privées ou publiques, ne répondant pas à plus de $25 \%$ des besoins, le reste était satisfait par des « gangs de commerce de sang », comme les nomme la presse égyptienne. Dans la même période, la police a arrêté, à plusieurs reprises, des bandes organisées qui achetaient du sang aux chômeurs et aux toxicomanes. Celles-ci ne se préoccupaient bien sûr pas de savoir si le sang était contaminé ou pas et n'adoptaient aucune mesure de sécurité mais prenaient le sang d'une personne tant que celle-ci était prête à le vendre, même tous les jours - ce qui a provoqué la mort ou l'hospitalisation de nombre de vendeurs de sang. Plusieurs personnalités médicales et politiques se sont publiquement exprimées sur ce sujet. Hamdî al-Sayyid, président de l'Ordre des médecins, a ainsi affirmé que :

[...] I'affaire dépasse le commerce du sang. Le marché noir est créé par une dangereuse crise de manque de sang [...] souvent on n'a de dons du sang que pendant les guerres [...]. Le fait de punir les vendeurs de sang ne va pas résoudre le problème, il y en aura d'autres [...]. La logique est de trouver une solution pour le problème [...]. Dans les années quatre-vingt, j'ai présenté un projet de loi à l'Assemblée du peuple, dont le principe est que chaque personne donnera du sang une fois dans sa vie, par exemple lorsqu'elle entre à l'université ou quand elle accède à une fonction, et que cela soit une condition pour avoir un permis de conduire [...] (Al-Wafd, 4 juin 1997)

À cette même époque, les gens qui ne pouvaient pas acheter de sang pour leurs proches malades, ou qui n'en trouvaient pas, donnaient leur propre sang, sans effectuer aucune analyse sanguine. En 1996, à la suite de plusieurs affaires de sang contaminé relatées par la presse égyptienne et après plusieurs enquêtes judiciaires, le ministère de la Santé décida de fermer quatre banques du sang après qu'il fut établi qu'une partie du sang qui en provenait contenait des virus d'hépatites et du sida. C'était une première dans l'histoire de l'Égypte.

Plusieurs personnes avec qui j'ai eu des entretiens m'ont affirmé avoir contaminé leurs proches sans le savoir. C'est ce que relate Ahmad:

Mon fils a subi une opération chirurgicale. Il avait besoin de sang, mais il n'y avait pas de sang disponible. On m'a demandé si un membre de la famille pouvait donner du sang, c'était urgent, j'ai dit : " moi je donnerai du sang, je suis son père, je suis la personne la plus proche, $c^{\prime}$ est normal que ce soit moi ». Quelque temps plus tard, j'ai appris que mon fils avait cette maudite maladie ; j'ai cherché ; j'ai voulu savoir comment mon fils avait eu ça. C'est un enfant que j'ai toujours protégé. Je n'ai pas trouvé la cause. Un jour, j'ai 
raconté au médecin que j'avais donné du sang à mon fils et que moi je ne suis pas malade. II m'a demandé de faire des analyses de sang et c'est là que j'ai découvert que j'avais ce virus et que c'est moi qui l'avais donné à mon propre fils. J'ai voulu sauver mon fils et je lui ai donné une maladie, une maladie très grave. Depuis, je déprime [...] j'essaie tout maintenant pour sauver mon fils.

Depuis la reconnaissance officielle de l'existence de l'hépatite C en Égypte, le ministère de la Santé a fait des efforts pour sécuriser la transfusion sanguine. Auparavant, dans les hôpitaux, un parent pouvait donner son sang directement et sans contrôle. Actuellement, et au moins pour ceux qui ne peuvent pas en acheter, on demande à la personne qui va subir une opération chirurgicale d'amener à l'hôpital des proches, afin que ceux-ci donnent une certaine quantité de sang. Le malade, en cas de besoin, sera transfusé avec le sang disponible et analysé à l'hôpital et le sang donné par la famille sera analysé et gardé pour d'autres malades. On procède ainsi à un échange de sang déjà analysé contre un sang qui va être analysé. C'est le principe qui a présidée à l'organisation des banques de sang aux États-Unis dans les années 1930 (Moulin, 1991).

Toutefois, malgré de telles précautions, et selon les médecins et la presse égyptienne, le contrôle de la transmission de la maladie est actuellement toujours loin d'être satisfaisant. Un jeune médecin déclare en effet :

Dans les milieux hospitaliers, la contamination continue, par exemple on ne fait pas attention, lors des accouchements, on n'utilise pas de gants et on utilise le même matériel pour plusieurs femmes. Plusieurs médecins et infirmiers ont attrapé cette maudite maladie de cette manière. Ni le personnel médical ni les patients ne sont à l'abri de ce fléau.

Un autre médecin m'a dit, concernant la prévention :

Le personnel n'est pas vraiment formé. Cela va du médecin aux agents d'entretien. Il faut voir comment on nettoie les salles d'opérations chirurgicales: on utilise des jets d'eau et des balais et on se débarrasse du sang, de la chair humaine, du coton [...] on sort ces choses de la salle, avec l'eau et le balai, et on continue d'étaler le sang avec le balai jusqu'à ce qu'on trouve des égouts. On nettoie des choses hautement contaminantes comme on nettoie une maison.

Un médecin affirme encore que le matériel utilisé pour les endoscopies n'est souvent pas fiable du point de vue du risque de contamination :

Nous avons beaucoup de malades et pas assez de matériel ni assez de temps. Lorsqu'on utilise ce matériel, il faut le stériliser vingt minutes, dans un produit spécial, pour qu'il ne transmette pas VHC, mais il y a des fois où nous ne disposons pas du temps nécessaire pour faire les choses comme il faut.

La presse évoque le manque d'incinérateurs et la manière entièrement dépourvue de sécurité dont les hôpitaux traitent leurs déchets, ce qui pose très clairement un problème de santé publique : 
Il faut que l'on réalise le danger que représentent ces déchets médicaux pour la santé publique. Il suffit de se promener autour des hôpitaux et de cabinets médicaux pour trouver des grandes quantités de poches de sang vides, des seringues, des médicaments, du coton et des pansements utilisés dont on s'est débarrassé, simplement en les jetant par terre [...] On trouve des jouets pour enfant, sans que le consommateur sachent si ces jouets peuvent ou ne peuvent pas contaminer les enfants avec des maladies contagieuses et dangereuses comme le virus de l'hépatite $C$ et le sida. Ils sont fabriqués à partir de produits de plastic dur qui proviennent de déchets hospitaliers. Il y a quelques semaines, on a confisqué de grandes quantités de seringues utilisées qui avaient été emballées comme des neuves et étaient destinées à être vendues dans les pharmacies.

Un autre moyen de contamination, dont on ne parle pas beaucoup en Égypte, est la toxicomanie. Si la contamination par le VHC dans les pays développés est due, à peu près, à 90 \% aux échanges des seringues entre toxicomanes, nous ne disposons pas de statistiques pour l'Égypte, bien que la toxicomanie par injection y soit présente. Le pays produit en effet de l'héroïne ; on désigne même un modèle de voiture Mercedes par le nom " al-bûdra », la poudre, indiquant par là que c'est l'argent gagné avec la vente de la « poudre » qui a permis aux propriétaires de ce modèle de l'acheter. Les drogues injectables existent bel et bien en Égypte, et les saisies de drogues ne manquent pas non plus ${ }^{3}$.

Le général Muhammad Farahât, chef des opérations au Département de la lutte anti-drogue, a déclaré - lors du $75^{\mathrm{e}}$ anniversaire de la création de celuici - que, sur le marché égyptien, le bângo, variété de cannabis cultivée en Égypte, représente $76 \%$ de l'ensemble des marchandises saisies, le cannabis $11 \%$, I'héroïne $5 \%$ (Al-Ahram Hebdo, 2 juin 2004). L'existence de drogues injectables implique l'existence de toxicomanes usant de seringues. Bien que nous ne possédions pas de statistiques sur le taux de toxicomanie en Égypte, le rapport d'Onusida estime que cinq consommateurs de drogues injectables sur dix utilisent des seringues non stérilisées (Onusida, 2006). Un responsable de I'ONG Caritas, qui travaille avec les enfants des rues toxicomanes, déclare qu'une grande majorité des toxicomanes réutilisent effectivement des seringues usagées. Mais il faut remarquer que, lorsqu'on parle de la contamination par injection chez les toxicomanes, c'est de la contamination par le sida dont il s'agit et non de la contamination par le virus de I'hépatite C. La prévention contre la contamination par I'hépatite $C$, chez les toxicomanes utilisant des seringues, n'est en effet prise en considération ni par les politiques de santé publique ni par les études scientifiques.

3. Un événement datant de 2003 a défrayé la chronique égyptienne : la confrontation musclée entre l'armée et une puissante famille de trafiquants de drogue de Haute-Égypte, où la police a dit avoir saisi une demi tonne de cannabis et cinquante kilos d'opium. 
Lorsque I'ampleur de la propagation du VHC en Égypte a été officiellement reconnue, les autorités égyptiennes ont fait savoir que l'État ne pouvait soigner les personnes atteintes par ce virus à cause du prix des médicaments. Le ministre de la Santé de l'époque a affirmé que le meilleur moyen de lutter contre cette maladie était la prévention et que son ministère allait s'y investir afin d'arrêter la contamination. Mais, jusqu'à présent, la transmission se poursuit; de nombreux spécialistes de la question affirment que tous les efforts nécessaires pour empêcher la contamination, particulièrement la contamination nosocomiale, n'ont pas été fournis. Lors d'un colloque organisé en Égypte sur cette question en octobre 2004, le président de l'Ordre des médecins - qui est aussi président du Comité de santé à l'Assemblée du peuple - a accusé les médecins de continuer à transmettre le virus eux-mêmes, à cause de leur négligence et de l'utilisation de matériel contaminé. Il a ajouté que le médecin qui effectue une opération chirurgicale et qui laisse le sang de son patient gicler sur son visage et sur ses mains peut attraper des virus et les transmettre à son tour à ses patients. Du reste, même les personnes qui ne connaissent pas le VHC se méfient de l'hôpital et de la négligence du corps médical. On dit souvent : «tu entres à l'hôpital avec une maladie et tu sors avec deux » ou encore "celui qui entre à l'hôpital est perdu et celui qui en sort vient de naître ».

Au demeurant, la forme épidémique particulière du VHC facilite la faiblesse de la mobilisation effective. Le VHC est bien une épidémie différente des autres. Le choléra, par exemple, était foudroyant ; il tuait massivement et rapidement et se propageait aussi rapidement, ce qui entraînait une mobilisation totale : politique, médicale, judiciaire, etc. (Delaporte, 1990). Ce n'est pas le cas avec le VHC, qui ne se transmet pas par simple contact personnel, qui tue lentement - les personnes atteintes pouvant même ne pas s'apercevoir qu'elles le sont - et pour lequel on ne dispose pas de statistiques indiquant le nombre de décès dont il est la cause. Certes, le virus est connu par la population, mais les modes de contamination ne le sont pas des personnes dont la pratique professionnelle peut favoriser celle-ci, comme les pédicures et les manucures, largement fréquentées par des hommes et, surtout, par les femmes. Durant mon enquête, je suis allée dans des salons de coiffure, dans les quartiers bourgeois comme dans les quartiers pauvres. On utilise les mêmes ciseaux pour tous les clients, que I'on se contente de laver avec un liquide mousseux. Lorsque j'ai dit aux esthéticiennes qu'on pouvait être contaminé par leur matériel, elles ont répondu que celui-ci était "lavé » et que personne ne leur avait jamais dit qu'il pouvait transmettre un quelconque virus. J'ai très rarement vu des clientes amener leur propre matériel. Cet exemple nous montre que, même au niveau de la simple prévention chez les professionnels, la déficience des autorités sanitaires est considérable.

En Égypte, les instances politiques ne se comportent pas avec l'épidémie du VHC comme il est de coutume en cas d'épidémie : ce n'est pas un 
événement mobilisateur et collectif mais un problème individuel. Les malades doivent se débrouiller comme ils peuvent, selon leurs moyens et l'étendue de leurs réseaux.

\section{LA MANIÈRE DONT LES MALADES SE SOIGNENT EN ÉGYPTE}

L'Égypte bénéficie d'une large tradition de médecine dite moderne qui remonte au dix-neuvième siècle ; elle a été établie par Clot-Bey, médecin et chirurgien français, chargé par Muhammad 'Alî d'en organiser son enseignement de 1824 à 1849. Pendant cette période, il a formé des médecins égyptiens et en a envoyé se former ou compléter leur formation en Europe (Moulin, 1990). Pour les Égyptiens, la biomédecine aujourd'hui est connue et reconnue, elle fait partie de leur histoire et de leur tradition médicale. On lui fait confiance, même si l'on se méfie des médecins; on sait que la médecine, lorsqu'elle est bien pratiquée, est le meilleur recours. On sait aussi que la médecine est mieux armée et mieux équipée dans d'autres pays, ainsi les patients qui en ont les moyens vont-ils se soigner en Europe ou au États-Unis. Le président de la République lui-même est allé se soigner en Allemagne. Lorsque des patients s'orientent vers d'autres thérapies, notamment "traditionnelles", ce n'est donc, le plus souvent, pas à cause de leurs croyances mais à cause de la méfiance qu'ils éprouvent vis-à-vis de la médecine telle qu'elle est pratiquée en Égypte ou parce qu'ils savent pertinemment que cette médecine ne peut rien leur offrir - surtout lorsqu'il s'agit d'une maladie chronique et coûteuse comme le VHC.

En un mot, le système de santé public égyptien n'a pas les moyens de gérer I'hépatite $\mathrm{C}$, du point de vue de la prévention comme de la cure. Il laisse donc à l'initiative privée la liberté de proposer des solutions palliatives. Pour mieux comprendre la situation, rappelons d'un mot ce que l'état offre en matière de soins, de manière générale. L'Égypte possède des cliniques (privées) qui vont du simplement correct (voire moins) au luxueux, des dispensaires, des hôpitaux universitaires, des hôpitaux relevant de la "sécurité sociale", des hôpitaux pour les militaires, d'autres pour la police, etc. L'offre est donc diversifiée, mais l'accès est lié à la place que l'on occupe dans la société, à l'existence d'accords professionnels, à des privilèges de fonction et au " piston ». L'Égypte dispose, certes, d'un système de sécurité sociale auquel $42 \%$ de la population adhère. L'adhésion à ce système est obligatoire pour tous les fonctionnaires, les employés du secteur public et les employés du secteur privé. Bénéficient aussi du système, les étudiants, les enfants de la naissance à l'âge de six ans, les retraités et les veuves d'anciens assurés. Les $47 \%$ de la population ne bénéficiant pas de la sécurité sociale peuvent encore se soigner aux frais de l'État, s'ils n'ont pas les moyens de se soigner à leurs frais dans le secteur privé.

Lorsqu' on entend des responsables du système de protection sociale égyptien en décrire le fonctionnement, on a l'impression qu'il est efficace et que 
les choses vont bien. Mais, en pratique, on se rend vite compte que bénéficier de la sécurité sociale n'implique pas nécessairement le droit de se soigner. Toute demande de soin y apparaît en effet complexe et tout service qu'on demande devient une exception, un "service », de sorte que l'on est bien soigné dans les hôpitaux de la sécurité sociale que lorsqu'on est " pistonné ». Les assurés sociaux ne peuvent pas choisir le médecin ou l'hôpital pour se soigner, ils sont obligés de recourir aux infrastructures appartenant à la sécurité sociale. Souvent, les malades expriment leur méfiance à l'égard des médecins qui en relèvent et évoquent les erreurs de diagnostics, le mauvais traitement des malades. Ahmad, un jeune employé, affirme :

J'ai la sécurité sociale, mais j'espère ne jamais avoir besoin d'y aller. Les médecins et les infirmiers traitent les malades comme des animaux. On dirait qu'on vient mendier ; c'est insupportable. Il faut attendre des heures et, en plus, ils peuvent te donner n'importe quel médicament.

Nadâ, une femme d'une cinquantaine d'années, diabétique et travaillant dans une école étrangère, raconte :

On ne peut pas vraiment compter sur la sécurité sociale. J'ai beaucoup souffert à cause de la sécurité sociale. Normalement, on doit me donner l'insuline, sinon je ne peux pas vivre. Je suis allée plusieurs fois la demander. On m'a demandé des certificats médicaux et plein d'autres papiers, mais chaque fois on me disait qu'il n'y avait pas de médicaments ou que le médecin n'était pas encore là ou on me disait de revenir un autre jour. Un fois, une amie m’a dit : "si tu veux avoir tes médicaments, il faut d'abord aller voir le médecin de la sécurité sociale dans son cabinet ». J'y suis allée et j'ai payé 80 Livres $^{4}$ la consultation, et après j'ai eu le médicament. Tu sais, il y a des fois où on me dit qu'il n'y a plus d'insuline et on me donne des cachets à la place. Quand je peux acheter mon médicament, je l'achète et, quand je ne peux pas, je prends les cachets, en attendant.

Néanmoins, malgré le mécontentement des usagers, les hôpitaux de la sécurité sociale restent le recours des classes défavorisées.

Les personnes qui se soignent aux frais de l'État - en dehors du système de protection sociale - se divisent en deux catégories totalement opposées : les favorisées, c'est-à-dire les personnalités ou les stars, qui sont envoyées aux frais de l'État dans les meilleurs hôpitaux européens ou américains, et les défavorisés, qui peuvent consulter des médecins en ne payant qu'une livre symbolique. Toutefois, pour cette seconde catégorie, lorsqu'il s'agit d'un grave problème de santé ou d'une opération chirurgicale, la situation se complique. La médecine gratuite a en effet mauvaise réputation, une réputation

4. Pour donner un ordre de grandeur, un instituteur égyptien gagne environ $200 \mathrm{LE}$ par mois au début de sa carrière. 
d'incompétence et de négligence, à cause du comportement désagréable de certains médecins et de certains infirmiers.

Fâtma a un fils qui doit subir une opération chirurgicale « aux deux oreilles ». Elle n'a pas les moyens de payer cette intervention. Elle est femme de ménage et son mari ne travaille pas. Elle amène son fils dans un hôpital universitaire, où sont dispensés les soins gratuits. À plusieurs reprises, on lui dit qu'il n'y a pas de place. La situation se prolonge ainsi pendant trois mois, jusqu'à ce qu'intervienne l'employeuse de sa fille qui téléphone à son neveu, professeur de médecine. Le fils est aussitôt admis à l'hôpital. Cependant, il faut pratiquer des radios ce qui, à cause du nombre des demandes, est apparemment difficile avant une semaine. Fâtma fait faire les radios ailleurs, en les payant. Avant I'opération, la famille donne du sang et achète le produit anesthésique. Après I'opération, il faut suivre un traitement pendant trois mois. Une partie du traitement est offerte par le professeur, qui explique à la mère qu'il fait cela «pour Dieu » et qu'il a l'habitude de faire de la charité de cette manière.

Une autre mère de famille $\mathrm{m}^{\prime}$ a raconté comment elle avait réussi à soigner sa fille :

La jambe de ma fille a enflé. Elle a eu très mal, je l'ai amené dans un hôpital à côté de chez moi, où j'ai payé seulement une Livre. Le médecin m'a dit que ce n'était pas grave et que ma fille avait seulement une fracture. II ne lui a pas fait de radios et il m'a envoyé chez le tamargî [homme à tout faire à I'hôpital : il nettoie, sert le thé...] qui a plâtré la jambe de ma fille. La jambe n'a pas arrêté d'enfler; ma fille n'a pas arrêté de pleurer ; elle a eu très mal. Deux jours après, je l'ai amenée chez un autre médecin, qui travaille aussi dans cet hôpital, mais je ne suis pas allée le voir à l'hôpital, je suis allée à son cabinet, où il faut payer 15 Livres. Tu sais, il vaut mieux aller au cabinet, parce qu'à I'hôpital les médecins sont toujours pressés, parce qu'ils ont beaucoup de monde et parce qu'ils veulent aller rapidement travailler dans leurs cabinets. Ils n'examinent pas bien à l'hôpital. Ce médecin m'a demandé de faire des radios. Ma fille avait une tumeur. Il a fallu faire une opération chirurgicale qui coûte 4000 Livres. Je ne pouvais pas payer cette somme. Le médecin m'a dit qu'on pouvait faire cette opération dans un hôpital de la mosquée en payant 1500 Livres. On a enlevé un os de la jambe de ma fille et, depuis, elle a une jambe qui est beaucoup plus maigre que l'autre. Tout cela est à cause du premier médecin qui lui a fait un plâtre...

Ces deux exemples montrent que s'il est toujours difficile d'accéder aux soins nécessaires et gratuits par l'intermédiaire du système de santé publique, il est possible d'accéder à ces mêmes soins, grâce à des réseaux interpersonnels et grâce à la pratique répandue de la charité. Aussi bien la charité individuelle que la charité organisée, celle des associations caritatives, substituent la providence privée à l'État-Providence. Mais paradoxalement, la charité privée s'appuie sur une infrastructure publique. En fait, le système public et le système privé coopèrent, de sorte que ni l'un ni l'autre ne constituent une 
véritable alternative. En d'autres termes, les deux se soutiennent réciproquement. II n'est pas plus possible, du point de vue du système privé, de rejeter le système public que, du point de vue du système public, de rejeter le système privé. Les demandeurs de soins sont donc rattachés aux deux et vont sans cesse de l'un à l'autre.

\section{ET COMMENT ILS SE SOIGNENT POUR LE VHC}

Après la reconnaissance officielle de l'existence de l'épidémie, le ministre de la Santé a encouragé les chercheurs égyptiens a mettre au point un médicament national à base de plantes, dont le prix serait à la portée de la population et à la portée de l'État, puisque celui-ci n'a pas les moyens de soigner tous les malades en recourant à l'Interféron. On estime que le coût de ce médicament peut s'élever jusqu'à 70000 Livres pour un patient et qu'il occasionnerait à l'État, s'il était distribué à tous les malades, une dépense de 70 milliards de Livres par an. D'après la presse, le ministre de la Santé aurait passé un accord avec l'une des plus importantes institutions de recherches du pays, le NRC (National Research Center) ${ }^{5}$. Cette démarche, une fois rendue publique, a favorisé, de manière directe ou indirecte, la multiplication des soins offerts sur le marché égyptien, aussi bien dans les pharmacies, chez les herboristes, chez des praticiens qu'aux abords des mosquées.

La position de départ du ministère - ne pas recourir systématiquement à I'Interféron et promouvoir la recherche d'un médicament à prix abordable - a été confortée par la polémique portant sur l'efficacité de l'Interféron en Égypte. Celle-ci est partie du fait que l'Interféron était considéré comme un remède faiblement efficace pour le génotype $4^{6}$, or le génotype 4 représente 90 \% des hépatites C en Égypte. La presse et de nombreux médecins égyptiens ont affirmé que l'Interféron n'était efficace contre le génotype 4 que dans 20 ou $30 \%$ des cas, et comportait, en outre, des effets secondaires très dangereux. Dans un article paru en 2004, dans le journal Al-'Arabî, il est fait état d'une étude de terrain :

En ce qui concerne l'Interféron, qui est le seul médicament disponible pour les malades du foie, l'étude a montré que ce médicament était une ruine pour l'économie nationale : le patient a besoin de trois injections par semaine, chacune coûte 1200 Livres et ce traitement dure six mois [...] À part son coût, la guérison n'est pas sûre. Et, comme l'a affirmé cette étude, le pourcentage de réussites du traitement par l'Interféron ne dépasse pas $50 \%$. Le pourcentage des rechutes, après six mois, est de moitié, c'est-à-dire que I'Interféron ne guérit à la fin que $25 \%$ des cas [...] Ses effets secondaires sont

5. Sur le NRC et sur les institutions de recherche, voir Radi, 2005.

6. À ce jour, six génotypes ont été identifiés. 
très dangereux pour la moelle sanguine et pour les cellules du foie [...] Les seuls qui profitent de ce traitement, comme a montré l'étude, sont quelques importateurs... (Al-'Arabî, 28 mars 2004)

Cette polémique a contribué à accréditer la position des autorités égyptiennes, puisque le remède trop cher y apparaît simultanément comme d'un effet limité et dangereux ${ }^{7}$. Elle justifie la recherche locale d'un palliatif. Comme les personnes atteintes par le VHC ne disposent pas d'un remède efficace et, à plus forte raison, qu'il n'existe pas en Égypte un protocole de soin bien établi, les patients doivent se soigner à l'aide de « remèdes» palliatifs prescrits par leur médecins, comme les anti-oxydants - qui, s'ils ne sont pas censés guérir la maladie, passent pour protéger le foie de la cirrhose et du cancer - ou encore la DDB, dite " pilule jaune », provenant de Chine, censée diminuer l'augmentation des enzymes hépatiques transaminases et permettre au foie d'améliorer ses capacités de renouvellement cellulaire. Un professeur de médecine, spécialiste de la question, m'a affirmé que moins de $10 \%$ de ses patients pouvaient se soigner avec I'Interféron et que, pour les autres, il était obligé de prescrire un médicament palliatif. C'est pour cela qu'il prescrit des anti-oxydants et la "pilule jaune », sans être sûr de leur efficacité.

Ainsi le coût du seul médicament connu et sa réputation de faible efficacité, la particularité du VHC 4 et la nécessité de proposer, malgré tout, des recours aux personnes atteintes ont-ils abouti à stimuler une production locale, pour une part soutenue par les instance politiques, pour une part issue d'initiatives privées, plus ou moins sérieuses, mais difficiles à mettre radicalement en cause - dans la mesure où elles découlent du retrait de l'État et de son choix assumé de promouvoir des solutions locales. La « découverte » par le National Research Center (NRC) d'un remède à base de plantes, la « découverte » par un médecin d'une cure utilisant des pigeons et la " découverte » d'un vétérinaire qui soigne ses patients avec du lait et de l'urine de chamelle sont autant d'exemples de soins palliatifs. Bien que très différents, ils se situent dans la même perspective et ne peuvent être opposés entre, d'un côté, une " découverte » relevant de la modernité, et, de l'autre, des « découvertes » relevant

7. Remarquons toutefois une nette évolution dans l'estimation de l'efficacité de I'Interféron. Si, durant une conférence organisée par le Centre culturel français du Caire, il y a quelques années, le Pr. Fontanet avait déjà estimé que $50 \%$ des patients égyptiens répondaient positivement au traitement, plus récemment, d'après une étude du même Pr. Fontanet et du Pr. Mustafâ K. Muhammad, 80 \% des personnes atteintes du VHC, dans la cohorte sur laquelle ils travaillent dans un village du delta du Nil, auraient répondu positivement au traitement PEG-IFNa (« Particularités de I'hépatite C en Égypte », Actualité de I'Inserm, n² 203, novembre 2006, www.insermactualités.com). 
de la tradition. Ces trois " découvertes», en effet, se présentent comme des solutions actuelles à une maladie actuelle, recourant indistinctement à des références crédibles disponibles.

\section{L'INVENTION DE CURES PALLIATIVES}

\section{La phytothérapie}

Le 7 juin 1995, le journal Al-Akhbâr annonçait que le NRC avait « découvert » un nouveau remède qui guérissait du VHC; d'autres journaux lui emboîtèrent le pas. Des malades se précipitèrent au NRC afin d'en acheter, mais il fallut attendre plusieurs jours pour y avoir accès. Il consistait en un paquet de plantes et un flacon de gouttes. Les plantes, que les patients devaient prendre par voie buccale, étaient censées protéger les cellules du foie et arrêter leur dysfonctionnement. Le flacon contenait des gouttes à instiller par voie nasale matin et soir, destinées à " détruire » le virus. Cette découverte représentait un grand espoir pour les malades ; le prix était, de plus, modéré et le médicament présenté comme totalement efficace et sans effets secondaires. Juste après l'annonce de cette " découverte », une femme nommée Gamîla porta plainte contre I' « inventeur » du médicament, le docteur 'Abd al-Bâsit, en affirmant que c'était elle qui lui avait confié les plantes et qu'elle était étonnée que son nom ne soit pas mentionné. Le président du NRC fit parvenir un démenti au premier journal qui avait fait état de la plainte de Gamîla :

Je suis fier de dire que le centre effectue des recherches [...] dans ce domaine et que les premiers résultats sont bons, prometteurs et donnent de l'espoir et que le centre continue ses recherches mais qu'il n'a annoncé aucun résultats. Tout ce qui se publie sur cette question, dans les différents médias, ne repose pas sur une réalité scientifique [...] En attendant la fin de ces recherches, le centre n'est responsable d'aucune déclaration provenant de personnes en son sein ou ailleurs [...] Monsieur le docteur 'Abd al-Bâsit n'a aucune relation avec le traitement de l'hépatite $C$ qui est un projet du National Research Center ... (Al-Akhbâr, 20 juin 1995)

Alors que se déroulait ce conflit complexe - Gamîla revendiquant l'invention du docteur 'Abd al-Bâsit et le président du NRC niant avoir travaillé sur ce médicament au centre - les patients se bousculaient pour acheter le médicament. Un malade qui s'en était procuré m'a raconté qu'au moment où celui-ci se vendait au Centre, il y avait à la fois des affiches qui vantaient la découverte et d'autres qui dégageaient la responsabilité du NRC.

Durant plusieurs mois, on discuta de l'efficacité du traitement. On parla du conflit des chercheurs, du manque de transparence financière, du complot des grandes sociétés qui importent l'Interféron. Lors d'une réunion, organisée en septembre 1995, sur les traitements à base de plantes à laquelle participèrent 
de nombreux chercheurs, le ministre de la Santé, le ministre de la Recherche scientifique et le président de l'Ordre des médecin, celui-ci attaqua le NRC en affirmant qu'il allait porter l'affaire du « médicament » devant les instances compétentes pour surveiller l'usage des deniers publics. En tant que président de l'Ordre des médecins, il accusait le NRC d'avoir expérimenté le traitement dans une unité médicale sans autorisation officielle. Puis il insista, avec le ministre de la Santé, sur la nécessité de prendre des dispositions urgentes pour appliquer les lois contrôlant l'usage des plantes et de leurs dérivés au médicament du NRC, afin de vérifier son efficacité (Al-Ahrâm, 24 septembre 1995). II s'est finalement avéré que le médicament n'était pas efficace.

\section{La thérapie par les pigeons}

Un autre traitement a été proposé, qui ne relevait pas de la phytothérapie ni d'aucune approche reliée à la "science ", bien qu'il fut découvert par un médecin. Ici, je me contenterai de traduire I'entretien d'un journaliste avec l'inventeur, le Dr. Muhammad al-'Abd, «spécialiste de médecine tropicale et de I'appareil digestif », qui prétendait possible de soigner l'hépatite C par les pigeons. Cette méthode consiste à mettre en contact l'anus d'un jeune pigeon femelle avec le nombril du malade, jusqu'au moment où le pigeon meurt. Actuellement, cette méthode est utilisée par plusieurs praticiens qui ne sont pas forcément des médecins. Le docteur Muhammad al-'Abd raconte :

Je suis spécialiste de l'appareil digestif et des maladies du foie. Je suis membre de I'Association des malades de l'appareil digestif et du foie et je suis membre des Association médicales américaine et européenne (sic). Je possède un magistère de médecine tropicale. Je traite les malades avec les médicaments pharmaceutiques reconnus, mais cette idée, je l'ai prise d'un bédouin du sud du Sinaï, il y a deux ans et demi. Je l'ai rencontré à Charm alChaykh, en 2002, alors que je participais à un colloque sur l'appareil digestif. En me promenant dans le désert, j'ai rencontré un vieux bédouin qui préparait du thé sur un feu de charbon. Je lui ai demandé un verre de thé. II m'a posé des questions sur la raison de ma présence dans la région. Je lui ai expliqué que je suis médecin et que je suis venu pour participer à un colloque scientifique, qui cherche du nouveau dans le traitement des maladies du foie. II m'a dit: " nous on soigne la jaunisse et le foie avec le petit pigeon qui n'a pas encore fait d'œuf, les gens guérissent sans aller chez le médecin ».

Au début, je n'étais pas convaincu, surtout en tant que médecin. Je sais parfaitement que le virus de l'hépatite $C$ ne se transmet que par le sang et, dans cette recette, je ne voyais rien qui indique que le patient transmet le virus à un pigeon. Le virus ne se transmet pas par l'attouchement. Je n'ai pas accepté cela, ni intellectuellement ni scientifiquement. Après mon retour, je me suis dit : " pourquoi ne pas essayer la recette de ce vieux monsieur, surtout qu'il n'existe pas de traitement efficace pour vaincre cette maladie et que le 
traitement est d'un prix exorbitant et sans résultats ». J'ai exposé la question à un patient qui a l'habitude de venir se soigner chez moi et il a accepté. Le pigeon est mort au bout de dix minutes. L'expérience s'est poursuivie quotidiennement, matin et soir, pendant dix jours, et chaque fois le pigeon mettait plus de temps pour mourir. Le dixième jour le pigeon est mort au bout de trois heures. J'ai demandé au patient de faire les analyses du HCV : le résultat était négatif. Je lui est demandé de faire les analyses du PCR (analyse de biologie moléculaire, qui recherche d'infimes traces du virus), qui est le moyen le plus fiable pour savoir si on a le VHC ou non et les résultats étaient négatifs, bien que le malade ait été atteint par le virus avant de faire cette expérience. Ensuite, j'ai fait la même expérience sur tous les patients qui venaient me consulter dans mon cabinet, à Damanhûr et à Alexandrie.

Lorsque je disséquais les nombreux pigeons morts après ces expériences, je voyais qu'il sortait de leur anus des cellules sanguines arachnoïdes qui vont dans une veine qui est reliée au foie du pigeon. Cela montre que ce qui se produit est une absorption des cellules qui se trouvent au nombril du patient par les cellules qui se trouvent dans l'anus du pigeon, qui ressemble à la saignée.

Jusqu'à présent, je ne peux pas expliquer ce qui se passe. Ça demande une recherche et des expériences scientifiques, mais je crois que le pigeon résorbe le virus du foie du malade, et cela demande d'analyser le sang et le foie du pigeon après sa mort, et si on y trouve le virus, c'est que le pigeon a absorbé le virus du malade et si on ne trouve pas de virus dans le sang et le foie, c'est qu'il existe une autre possibilité - c'est que le pigeon lui-même possède des glandes spécifiques dans l'anus, qu'il s'en dégage quelque chose, lorsqu'il touche le nombril du patient et qui attaque le VHC dans le foie et le détruit [...] Je possède trois cabinets médicaux, où je gagne beaucoup d'argent et je fais partie d'une famille très fortunée. Je suis l'un des plus gros contribuables de la ville de Damanhûr [...] Je ne me fais payer que la consultation. N'importe qui peut soigner de cette manière, y compris les malades eux-mêmes, sans l'intervention d'aucun médecin, sauf pour les analyses, pour être sûr de leur guérison ou non. Moi, je ne possède ni laboratoire d'analyses du sang ni pigeonnier pour profiter de ce traitement... (Al-Musawwar, 9 janvier 2004)

Le ministère de la Santé a fermé les deux cabinets de ce médecin et plusieurs personnes pratiquant cette méthode de soin ont été arrêtées. Mais le traitement est toujours répandu et pratiqué par d'autres personnes, qui n'ont besoin, pour ce faire, ni d'être médecin ni de posséder un cabinet médical. Le principe de crédibilité retenu ici est simple : il réside dans l'affirmation de l'efficacité d'un remède "traditionnel », livré par un "vieux bédouin ». Le médecin atteste cette efficacité par son impuissance même à la comprendre et à la réfuter. En racontant qu'il ne comprend pas mais qu'il fait un constat, il donne finalement un argument sérieux pour croire qu'il est possible de se soigner par l'imposition d'un anus de pigeon. 


\section{La cure par le lait et l'urine de chamelle}

Le docteur Zaynab al-Sunûsî est vétérinaire à Marsâ Matrûh, ville balnéaire à l'ouest d'Alexandrie. Elle soigne l'hépatite $C$ par le lait et l'urine de chamelle. Suivant ce traitement, il suffit de prendre un verre de lait et une cuillère d'urine de chamelle pendant quelques semaines pour guérir de l'hépatite C. Elle a fondé sa découverte sur un hadith rapportant que selon le Prophète le lait de chamelle guérit de la jaunisse ou des maladies de foie. Les malades se sont précipités chez elle, venant de toute l'Égypte. Cette nouvelle "découverte ", d'autant plus attractive qu'elle était liée à une tradition prophétique, a donné lieu à plusieurs publications qui décrivent en détail les conditions dans lesquelles le Prophète a conseillé le lait de la chamelle ainsi que toutes ses vertus. Elles s'attachent à justifier et à renforcer les conseils du Prophète, en évoquant des " recherches scientifiques » qui auraient été conduites en Israël, à Doubaï, en Arabie Saoudite ou encore en Libye. Cette cure bénéficie de la crédibilité accordée à la référence religieuse et se fonde sur l'idée que toute science est déjà dans le Coran. Il existe, au demeurant, des livres qui décrivent la médecine du Prophète. L'un de ces nombreux ouvrages s'intitule d'ailleurs La guérison avec le lait et l'urine du chameau: sunna prophétique et miracle médical's. Il a été publié avec l'aval de l'université d'al-Azhar, qui a attesté qu'il ne comportait pas d'éléments contraires à l'Islam et qu'il n'y avait donc pas d'objection à sa publication. Cette référence fait échapper le docteur Zaynab al-Sunûsî à l'accusation de charlatanisme.

À la suite de la publicité faite au remède, le prix du lait de chamelle a considérablement augmenté. Partout au Caire, on distribue des brochures qui présentent les bienfaits de ce lait et le nombre considérable de maladies qu'il peut guérir. Sur les brochures, tout est imprimé sauf le numéro de téléphone et le nom de la personne à contacter pour acheter le lait - ceux-ci sont écrits à la main et changent d'une série de brochures à l'autre.

Toutefois, au-delà de la seule crédibilité du remède, il faut surtout tenir compte du désir des malades de trouver une cure efficace pour leurs maux. C'est ce désir, plus que la crédibilité, qui enclenche les itinéraires thérapeutiques :

J'essaie tout ce que je peux. Pourquoi pas le lait et l'urine de chamelle?

De toute façon, dans l'état où je suis, s'ils ne me font pas de bien, ils ne peuvent pas me faire du mal. Il faut croire à tout et je vais essayer tout ce qui est à ma portée. II faut croire, je ne vais pas rester sans rien faire et de toute façon le lait est conseillé par notre Prophète.

8. Écrit par un certain Chihâb al-Badawî Yassî, et édité par Maktabat minhâj alnubuwwa en 2003 (seconde édition). 


\section{UN SYSTÈME PALLIATIF}

Dans les faits, le système médical égyptien ne paraît pas à même de lutter efficacement contre l'épidémie, ni par des mesures de prévention ni en finançant la recherche d'un traitement. De plus, il est largement responsable du fait épidémique. C'est ce constat des autorités médicales égyptiennes qui ouvre la porte à la formulation de solutions palliatives, privées ou publiques, présentées comme des "découvertes".

Ces découvertes sont bien des expressions de la " modernité » et non de simples résurgences de la «tradition ». Elles se relient à un problème contemporain et s'adressent, d'ailleurs, à des hommes " modernes ». La production de ces traitements est reliée à des systèmes de références crédibles dont on use généralement de manière conjointe comme l'autorité coranique, celle de la « tradition » et, finalement, dans chacun des cas rapportés, I'autorité de I'épreuve scientifique. Ne pouvant rester absent de l'espace public dans ce domaine, le système de santé public égyptien a participé à la production de traitements en tentant de promouvoir une phytothérapie. Nous n'avons donc pas affaire ici à la résurgence de croyances anciennes mais à la production commune d'un système pluraliste fondé sur des références qui ne s'excluent pas et, surtout, ne sont en rien un refus de la modernité scientifique. Ce système est hybride et néanmoins cohérent en tant que ressource collective palliative découlant de l'incapacité de l'État égyptien à promouvoir et maintenir un système de santé public efficace. Comme à l'accoutumée, cette instance politique, si elle est incapable d'apporter une solution globale, propose des solutions partielles dont les gens peuvent tirer partie, d'une manière ou d'une autre. Ainsi la solution proposée par l'État égyptien est-elle suffisamment « impliquante » pour, à la fois, atténuer et disséminer les critiques : si on ne peut pas se soigner avec I'Interféron, on peut espérer que d'autres médicaments seront accessibles. C'est ce que dit l'homme qui se soigne au lait et à l'urine de chamelle : « je vais essayer tout ce qui est à ma portée ».

Plus globalement, l'État n'a pas les moyens de généraliser une couverture sociale efficace - qui permettrait, par exemple, de faire bénéficier les malades de I'Interféron - mais les quelques avantages sociaux qu'il procure comme les infrastructures qu'il met en place peuvent profiter aux démunis, dans des formules alliant le clientélisme et la charité. Les itinéraires thérapeutiques découlent donc d'abord des palliatifs offerts dans une situation où les ressources sont difficiles, où l'on fait souvent avec les moyens du bord. Ces palliatifs joints à l'évidence de la faiblesse des moyens de l'Égypte expliquent que, pour une large part, l'épidémie de l'hépatite $C$ n'ait pas donné lieu à une protestation sociale, comme c'eût été le cas en Europe si les instances politiques s'étaient montrées si peu capables de réparer les conséquences dramatiques de leur carences. On a assisté en Égypte, au contraire, à une sorte de privatisation de la réponse à l'épidémie de I'hépatite $C$. 


\section{RÉFÉRENCES BIBLIOGRAPHIQUES}

Delaporte F., 1990, Le Savoir de la maladie. Essai sur le choléra de 1832 à Paris, Paris, PUF.

Dodier N., 2003, Les Leçons politiques de l'épidémie de Sida, Paris, Éditions de l'EHESS.

Fassin D., 1996, L'Espace politique de la santé, Essai de généalogie, Paris, PUF.

Hours B., 2001, "Pour une anthropologie de la santé en sociétés », dans B. Hours (dir.), Systèmes et politiques de santé. De la santé publique à l'anthropologie, Paris, Karthala.

Moulin A.M., 1990, "Les Arabes, les Turcs et la Révolution Française. Révolutions médicales et révolutions politiques en Égypte (1865-1917)», Revue de la Méditerranée et du Monde musulman, n 52-53.

- 1991, Le Dernier voyage de la médecine, Paris, PUF.

Onusida, 2006, Moyen Orient et Afrique du Nord : le point sur l'épidémie du sida.

RADI S., 2005, "L'état de la science en Égypte ", Études et documents du Cedej, $\mathrm{n}^{\circ} 18$.

- 2006, "Débats de presse, scandale et mise en place d'une politique de prévention : à propos de I'hépatite C en Égypte ", Revue d'épidémiologie et de santé publique. 